\title{
Two successful pregnancies achieved by converting an in vitro fertilization cycle to an intrauterine insemination cycle in five cases with documented premature ovulation
}

\author{
Kubilay Vicdan, Cem Akarsu, Eran Sözen, Burcu Buluç, Deniz K. Üstündağ, Kutay Biberoğlu \\ Private Ankara IVF Center, Ankara, Turkey
}

\begin{abstract}
We here report two successful pregnancies obtained by converting an in vitro fertilization (IVF) cycle to an intrauterine insemination (IUI) cycle in five poor responder patients whose oocyte pick-up (OPU) procedures were canceled due to documented premature ovulation immediately before OPU. To our knowledge, this is the first article that demonstrates that switching an IVF cycle to an IUI cycle when premature ovulation occurs on the day of OPU can produce successful pregnancies, even in poor responder patients. (J Turk Ger Gynecol Assoc 2016; 17: 233-5)
\end{abstract}

Keywords: In vitro fertilization, intrauterine insemination, premature ovulation, poor response

Received: 14 June, 2016 Accepted: 27 October, 2016

\section{Introduction}

Diminished ovarian reserve (DOR) is closely associated with poor response to ovarian stimulation, detection of premature luteinizing hormone (LH) surge before ovulation trigger, premature ovulation, and failure to retrieve oocytes during timed oocyte pick-up (OPU) procedures $(1,2)$. It is a common and accepted strategy to cancel the cycle, to proceed to egg collection, or to convert the in vitro fertilization (IVF) cycle to an intrauterine insemination cycle (IUI) in these poor responder patients (2-6); however, it is very challenging for the clinician to decide on a course of action in cases when unpredicted, premature ovulation is discovered as an unwelcome surprise immediately before a timed OPU. Although cycle cancelation, puncture of smaller residual follicles, and aspiration of follicular fluid from the pouch of Douglas are recommended methods, only a few successful pregnancies have been reported with these attempts (7).

Therefore, we adopted a different strategy: performing lastminute transvaginal sonography (TVS) before anesthesia to be certain whether ovulation has occurred; if premature ovulation has occurred, the IVF cycle is changed to a rescue IUI in couples who have at least one patent fallopian tube and a total progressive sperm count of $>5$ million. Here, we report the cycle outcomes of five patients managed with this approach.

\section{Case Presentation}

In this article, the results obtained from five consecutive poor responder patients undergoing IVF complicated by premature ovulation are evaluated. The demographic and cycle characteristics of the patients are summarized in Table 1. In all cases, ovarian stimulation was started on day 2 of the cycle using recombinant or urinary follicle-stimulating hormone (FSH) or human menopausal gonadotropin (hMG) in conjunction with gonadotropin-releasing hormone $(\mathrm{GnRH})$ antagonist. A starting dose of 300 to $450 \mathrm{IU} / \mathrm{d}$ was administered according to the patient's age, ovarian reserve, and previous cycle response. A TVS scan was performed on cycle day 5, and GnRH antagonist was started on day 6 in all cases as a fixed protocol. The gonadotropins and antagonist were both continued until the leading follicles reached $\geq 17 \mathrm{~mm}$, at which time ovulation was triggered with $10.000 \mathrm{IU}$ urinary hCG (Pregnyl, MSD) or $500 \mu \mathrm{gr}$ recombinant human chorionic gonadotropin (hCG) (Ovitrelle, Merc Serono); the OPU procedure was scheduled for 35 hours later. On the day when OPU was scheduled, lastminute TVS before anesthesia detected no follicles in both ovaries in these cases, and a diagnosis of premature ovulation was made. Instead of canceling the cycle or attempting to puncture the remaining follicular residues, IUI was offered and performed as a rescue treatment after obtaining written consent from the couples. Semen specimens were prepared 
Table 1. Demographic features and cycle characteristics of the patients

\begin{tabular}{|c|c|c|c|c|c|}
\hline & 1 & 2 & 3 & 5 & 6 \\
\hline Female age/years & 25 & 39 & 37 & 37 & 38 \\
\hline Infertility duration/years & 6 & 1.5 & 7 & 13 & 17 \\
\hline FSH (mIU/mL) & 11.7 & 21.1 & 20.1 & 9 & 11 \\
\hline Antral follicle count & 2 & 3 & 3 & 4 & 4 \\
\hline HSG, patent tube & + & + & + & + & + \\
\hline TPMS/swim up (million) & 6.48 & 6.64 & 21 & 6.23 & 16.2 \\
\hline Infertility reason & Unexplained & Unexplained & Unexplained & Unexplained & Unexplained \\
\hline Previous OI cycle & - & 6 & - & - & - \\
\hline Protocol type & Antagonist & Antagonist & Antagonist & Antagonist & Antagonist \\
\hline Gonadotropin type & uFSH & $\mathrm{uFSH}+\mathrm{HMG}$ & HMG & r-FSH & HMG \\
\hline Starting dose & 300 & 375 & 450 & 375 & 375 \\
\hline Total gonadotropins (Units) & 2625 & 2550 & 4650 & 3375 & 6825 \\
\hline Trigger day & 11 & 11 & 14 & 10 & 13 \\
\hline Follicle number, hCG day & 1 & 1 & 2 & 4 & 2 \\
\hline Follicle number at pick-up & - & - & - & - & - \\
\hline Pregnancy & - & - & - & + & + \\
\hline
\end{tabular}

FSH: follicle-stimulating hormone; TPMS: total progressive motile sperm; OI: ovulation induction; IUI: intrauterine insemination; IVF: in vitro fertilization; hCG: human chorionic gonadotropin; HMG: human menopausal gonadotropin; HSG: hysterosalpingogram

by density gradient centrifugation. All cases received luteal support with vaginal micronized progesterone. A blood test for hCG was performed 14 days after IUI in all 5 cases, and 2 had positive results. Transvaginal ultrasound confirmed a clinical pregnancy at 7 weeks of gestation in both patients, and both of these pregnancies are currently ongoing after 20 weeks.

\section{Discussion}

A meta-analysis has demonstrated that women with antral follicle counts of fewer than four were 8.7 times less likely to become pregnant after IVF and 37 times more likely to have their cycle canceled compared with normoresponders (8). In poor responders with stimulated IVF cycles, the decision whether to cancel the cycle, to continue with the egg collection, or to convert to an IUI cycle is very challenging. Matorras et al. (4) assessed the utility of transforming an IVF cycle with low response to an IUI cycle in 57 patients undergoing IVF due to unexplained infertility, mild to moderate male factor, or previous IUI failure. They reported a pregnancy rate of $14 \%$ in IVF poor responders with at least 2 to 4 follicles; they concluded that IUI should be considered in the management of poor responders. Contrastingly, in another study of 1350 IVF cycles with one or two mature follicles, conversion of an IVF cycle to an IUI cycle was reported to have the poorest prognosis, while proceeding with egg collection was much more successful in achieving pregnancy (2). Another multicenter comparative study which analyzed 7176 initiated cycles also suggested that IVF should be pursued for women demonstrating two follicles, while conversion to IUI was recommended for cycles with only one follicle, if sperm and tubal parameters were favorable (3). It was also clearly demonstrated that performing IUI as a rescue treatment provided no advantage over taking no further action when no oocytes were collected during a timed follicular puncture (6).

Encountering unexpected premature ovulation at the time of OPU under anesthesia is another challenge for the clinician as well as for the infertile couple; this is a totally different situation with very few treatment alternatives. Wu et al. (7) reported three cases of successful pregnancies with puncture of small or medium-sized follicles to retrieve oocytes (in vitro maturation was performed in two cases); however, this remains the only success story which was not reproduced by others. Furthermore, puncture of the remaining follicles and aspiration of the peritoneal fluid necessitate additional methods, such as OPU itself, and anesthesia; this imposes additional financial and emotional burdens on the couple. Cancellation of the 
cycle is a more logical approach in the case of documented premature ovulation during a timed OPU procedure because it is impossible to detect the exact time of ovulation or to know if the oocytes have already been captured by the Fallopian tubes. In this article, our results demonstrate that couples with at least one patent tube and with a normal sperm count can benefit from a rescue IUI in the case of premature ovulation detected at the time of OPU, even after previous failed IUI or IVF treatment cycles. Therefore, we suggest a "rescue" IUI as an alternative approach in unexplained infertility cases with DOR who are scheduled to undergo IVF and who experience premature ovulation before a timed OPU procedure.

Ethics Committee Approval: N/A.

Informed Consent: Written informed consent was obtained from patients who participated in this study.

Peer-review: Externally peer-reviewed.

Author Contributions: Concept - C.A.; Design - K.V.; Supervision - K.B.; Resources - K.V.; Materials - C.A.; Data Collection and/or Processing - E.S., B.B.; Analysis and/or Interpretation - K.V.; Literature Search D.K. U.; Writing Manuscript - K.V.; Critical Review - K.B.

Conflict of Interest: No conflict of interest was declared by the authors.

Financial Disclosure: The authors declared that this study has received no financial support.

\section{References}

1. David ER, Zakarin L, Chao K, Meyer L, Owen KD, Rosenwaks Z. Diminished ovarian reserve is the predominant risk factor for go- nadotropin-releasing hormone antagonist failure resulting in breakthrough luteinizing hormone surges in in vitro fertilization cycles. Fertil Steril 2014; 102: 99-102.

2. James DM, Nicopoullos MD, Abdalla H. Poor response cycles: when should we cancel? Comparison of outcome between egg collection, intrauterine insemination conversion, and follow-up cycles after abandonment. Fertil Steril 2011; 95: 68-71.

3. Quinquin M, Mialon O, Isnard V, Masin M, Parinaud J, Delotte J, et al. In vitro fertilization versus conversion to intrauterine insemination in Bologna-criteria poor responders: how to decide which option. Fertil Steril 2014: 102: 1596-601.

4. Matorras R, Corcostegui B, Mendoza R, Ramon O, Aporicio V, Uriarte MT, et al. Converting an IVF cycle to IUI in low responders with at least 2 follicles. J Reprod Med 2003; 48: 10: 789-91.

5. Sönmezer M, Pelin Cil A, Atabekoğlu C, Ozkavukçu S, Ozmen B. Does premature luteinisation or early surge of LH impair cycle outcome? Report of two successful pregnancies. J Assist Reprod Genet 2009; 26: 159-63.

6. Matorras R, Aparicio V, Corcostegui B, Prieto B, Mendoza R, Ramon $\mathrm{O}$, et al. Failure of intrauterine insemination as a rescue treatment in low responders with adequate HCG timing with no oocytes retrieved. Reprod Biomed Online 2014; 29: 634-9.

7. Wu FS, Lee RK, Hwu YM. Encountering premature ovulation during controlled ovarian hyperstimulation in IVF/ICSI cycles. Taiwan J Obstet Gynecol 2012; 51: 2: 256-9.

8. Gibreel A, Maheshwari AM, Bhattacharaya S, Jhonson NP. Ultrasound tests of ovarian reserve: a systematic review of accuracy in predicting fertility outcomes. Hum Fertil 2009; 12: 95-106. 